\title{
Prevenção quaternária e a redução do fluxo de encaminhamentos à angiologia e pedidos de ultrassonografia Doppler
}

\author{
Quaternary prevention and reduction in the flow of referrals to angiology and the Doppler \\ ultrasonography requests
}

\section{Prevención cuaternaria y la reducción del flujo de referencias a angiología y pedidos de ultrasonografía Doppler}

\author{
Eduardo Simon ${ }^{1 \oplus}$, Denise Mota Araripe Pereira Fernandes ${ }^{2 \oplus}$, Lana Muriely Borges de Assis $^{2 \oplus}$, Melissa Maria Medeiros de Morais $^{2 \oplus}$, \\ Anne Thaísa Dantas Carvalho ${ }^{2 \oplus}$, Marina de Lima Pinheiro ${ }^{2}{ }^{\circledR}$, Kerlonny Fopsy ${ }^{\circledR}$ \\ ${ }^{1}$ Universidade Federal da Paraíba (UFPB) - João Pessoa - PB. \\ 2 Faculdade de Ciências Médicas da Paraíba / Secretaria de Saúde de João Pessoa - PB.
}

\section{Resumo}

Introdução: A iatrogenia é uma causa importante de morbimortalidade, logo, a prevenção quaternária (P4), ação que ceifa a cascata diagnóstica supérflua, foi incluída como exercício de boa prática. A medicina de família e comunidade (MFC) introjeta a P4 na vivência, sendo capaz de usar ciência aliada a habilidades de comunicação para conhecer as pessoas, resolvendo assim cerca de $85 \%$ das queixas, o que inclui as de origem vascular. Existe uma alta demanda no Sistema Único de Saúde (SUS) tanto na marcação de exames quanto no encaminhamento para especialistas focais no que tange às queixas circulatórias, mas nem sempre encaminhar é oportuno. Nesse cenário, a MFC funciona como um caminho para a P4. Objetivos: O objetivo do presente estudo foi avaliar as referências para cirurgia vascular e angiologia e as solicitações de ultrassonografia (USG) Doppler de vasos de unidades de saúde da família (USFs) de João Pessoa/PB, analisando as taxas de solicitações por população adulta (>18 anos) adscrita, nas unidades conveniadas ao programa de residência em medicina de família e comunidade (PRMFC) há mais de 1 ano, há menos de 1 ano e naquelas sem convênio com PRMFC, sob o olhar crítico da P4. Métodos: Trata-se de um estudo transversal a partir de dados fornecidos pela Central de Regulação da Secretaria Municipal de Saúde de João Pessoa (SMS/JP), entre janeiro de 2017 e agosto de 2018. Para realização dos cálculos de correlação entre as variáveis estudadas, utilizou-se o teste de Kruskall-Wallis através do software SPSS 21.0. Resultados: O número total de pedidos de exames em análise foi de 3.918 , oriundos de 90 USF, que somam uma população adscrita maior de 18 anos de 332.819 pessoas. Solicitouse 1 Doppler a cada 85 habitantes adultos. Os resultados do estudo sugerem que os médicos residentes solicitam mais exames que os médicos não residentes, mas por outro lado apresentam menor taxa de encaminhamento aos especialistas. Conclusões: Estes achados podem sugerir que os médicos residentes encaminham menos, o que pode estar relacionado ao aumento da resolubilidade da atenção primária à saúde (APS) e ao ensino da P4 sob orientação de um médico de família e comunidade preceptor. Entretanto, novos delineamentos de pesquisa são necessários para melhor elucidar esta hipótese.

Palavras-chave: Atenção Primária à Saúde; Ultrassonografia Doppler; Internato e Residência; Medicina de Família e Comunidade.

Como citar: Simon E, Fernandes DMAP, Assis LMB, Morais MMM, Carvalho ATD, Pinheiro ML, Fopsy K. Prevenção quaternária e a redução do fluxo de encaminhamentos à angiologia e pedidos de ultrassonografia Doppler. Rev Bras Med Fam Comunidade. 2021;16(43):2572. https://doi.org/10.5712/rbmfc16(43)2572
Autor correspondente:

Eduardo Simon.

E-mail: esimon81@gmail.com

Fonte de financiamento:

declaram não haver.

Parecer CEP:

CAAE № 02954918.0.0000.8069

Procedência:

não encomendado.

Avaliação por pares:

externa.

não encomendado.

Recebido em: 15/06/2020.

Aprovado em: 22/05/2021. 


\begin{abstract}
Introduction: latrogenesis is an important cause of morbimortality and quaternary prevention (P4), an action that reduces the superfluous diagnostic cascade, was included as an exercise of good practice. Family practice projects P4 into practice, being able to use science combined with communication skills to know people, solving about $85 \%$ of complaints, including vascular discomforts. There is a high demand at Sistema Único de Saúde (SUS) both in scheduling exams and referrals to focal specialists with regard to circulatory complaints, but referring is not always appropriate. In this scenario, family practice works as a pathway to P4. Objectives: The aim of the present study was to evaluate the referrals for vascular surgery/angiologist and requests for Doppler ultrasonography (USG) of vessels from family health units (USF) in João Pessoa, PB, analyzing the rates of requests per registered adult population (>18-years-old) in the units affiliated to the family practice medical residency program (FPMRP) for more than a year, less than a year and in the non-affiliated ones, under P4's critical view. Methods: This is a cross-sectional study of data provided by the Regulation Center of the Municipal Health Department of João Pessoa, between January 2017 and August 2018. To perform the correlation calculations between the studied variables, Kruskall-Wallis' correlation test was used through the SPSS 21.0 software. Results: The total number of exam requests under analysis was 3,918, from 90 USF, which add up to an enrolled population over 18-years-old of 332,819 people. For every 85 adult inhabitants, one Doppler exam was requested. Data suggest that the resident doctors request more exams than the non-residents, but on the other hand they have lower rates of referrals to focal experts. Conclusions: These findings may suggest that resident physicians refer less, which may be related to the increase in the resolution of primary care and the teaching of P4 under the guidance of a family practice preceptor. However, new research designs are considered to better elucidate this hypothesis.
\end{abstract}

Keywords: Primary Health Care; Ultrasonography, Doppler; Internship and Residency; Family Practice.

\title{
Resumen
}

Introducción: La iatrogenia es una causa importante de morbilidad y mortalidad, por lo que se incluyó como ejercicio de buena práctica la prevención cuaternaria (P4), una acción que corta la cascada diagnóstica superflua. La medicina familiar y comunitaria (MFC) introduce la P4 en la práctica, siendo capaz de utilizar la ciencia aliada a las habilidades de comunicación para conocer a las personas, resolviendo así alrededor del $85 \%$ de las quejas, lo que incluye las quejas vasculares. En el Sistema Único de Salud (SUS) brasileño hay una gran demanda, tanto para programar exámenes como para derivar a los especialistas focales las dolencias circulatorias, pero la derivación no siempre es oportuna. En este escenario, la CBM funciona como una vía hacia la P4. Objetivo: El objetivo del presente estudio fue evaluar las referencias de cirugía vascular y angiología y las solicitudes de ultrasonografía Doppler (USG) de vasos de las unidades de salud de la familia (USF) de João Pessoa/PB, analizando las tasas de solicitudes por población adulta (>18 años) asignada, en las unidades asociadas al programa de residencia en medicina de la familia y de la comunidad (CFMRP) por más de 1 año, por menos de 1 año y en las que no tienen convenio con el CFMRP, bajo la visión crítica del P4 Métodos: Se trata de un estudio transversal de datos proporcionados por la Central de Regulación de la Secretaría Municipal de Salud de João Pessoa (SMS/JP), entre enero de 2017 y agosto de 2018. Se utilizó la prueba de Kruskall-Wallis para realizar los cálculos de correlación entre las variables estudiadas mediante el programa informático SPSS 21.0. Resultados: El número total de solicitudes de pruebas analizadas fue de 3.918, procedentes de 90 USF, que suman una población asignada mayor de 18 años de 332.819 personas. Se solicitó un Doppler por cada 85 habitantes adultos. Los resultados sugieren que los médicos residentes solicitan más pruebas que los no residentes, pero por otro lado tienen una tasa de derivación a especialistas más baja. Conclusiones: Estos resultados pueden sugerir que los médicos residentes derivan menos, lo que puede estar relacionado con la mayor resolutividad de la Atención Primaria de Salud (APS) y la enseñanza de la P4 bajo la dirección de un médico de familia y un preceptor comunitario. Sin embargo, se necesitan nuevos diseños de investigación para dilucidar mejor esta hipótesis

Palabras clave: Atención Primaria de Salud; Ultrasonografía Doppler; Internado y Residencia; Medicina Familiar y Comunitaria.

\section{INTRODUÇÃO}

A iatrogenia tem despertado crescente atenção dos pesquisadores, visto ser uma importante causa de morbimortalidade no mundo. ${ }^{1}$ Cada vez mais, percebe-se a urgência de considerá-la com mais criticidade ante os processos de saúde e adoecimento. Tal observação leva à consequente adição do conceito de prevenção quaternária ( $\mathrm{P} 4)$ no rol das atividades que se apresentam como estratégias transversais e potentes de cuidado. Com o objetivo de evitar medidas médicas fúteis, tanto a nível diagnóstico como terapêutico, a P4 caminha para além da prevenção, visto ser inclusiva, não reducionista, integrada e biopsicossocial ao englobar esferas humanas, sociais e políticas, acolhendo a incerteza e não a mascarando sob a égide do senso comum. ${ }^{2}$ Além de denunciar e prevenir a naturalização e medicalização de situações como fome, desigualdade, violência, racismo e exploração. Assim, é premente a necessidade de ensino e divulgação nos níveis populacionais e acadêmicos da $P 4^{3,4}$ 
Em geral, quanto menos dialogam com seus pacientes, mais os médicos tendem a solicitar exames complementares. Este fenômeno pode levar a sérios e imprevisíveis efeitos nocivos. ${ }^{5}$ Além de empobrecer a dimensão relacional entre médicos e pacientes, o próprio raciocínio clínico unidirecional e hiperfocalizado, por vezes feito por especialistas, pode ser assimétrico: por um lado, tendem a apresentar responsabilidade reduzida para com o conjunto dos problemas de saúde dos pacientes, por outro, dedicam atenção demasiada às patologias do escopo especializado, em detrimento do todo. A esta desconsideração do "todo pela parte", soma-se uma busca intensa por acurácia diagnóstica, que pode conduzir à justificação de intervenções agressivas, que as especialidades focais proclamam altivo contraponto em relação à prática generalista. ${ }^{1}$

A medicina de família e comunidade (MFC), por sua vez, adota a abordagem de compreender como as pessoas entendem o significado de ter saúde, como percebem a doença e quais as dimensões da experiência do adoecimento, ajudando, desta forma, a superar a visão reducionista das patologias. Isto instrumentaliza os profissionais de saúde a considerar também os contextos psicossociais ampliados, que influenciam as diversas etapas do processo saúde-doença. Essa metodologia de condução clínica requer competência profissional para garantir um cuidado integral e resolutivo. ${ }^{6}$

Neste sentido, uma das funções do médico de família consiste no exercício da P4, a qual parte do compromisso tanto com o cuidado quanto com o ofício. É peça do contrato social implícito entre a profissão médica e a sociedade, no qual está delegada àquela o atributo de grandes poderes e responsabilidades, em troca de que realize o que está a seu alcance dentro das premissas éticas e científicas. ${ }^{1}$

O conhecimento das doenças mais prevalentes na comunidade é fundamental para uma atuação de qualidade na Atenção Primária à Saúde (APS), pois permite a resolução de até $85 \%$ das queixas avaliadas. Ao conhecimento médico, deve ser agregada à capacidade de conhecer a pessoa. Tal aptidão é indispensável ao profissional que atua neste nível de atenção. ${ }^{7}$ É de forma integrada e singular que se deve abordar queixas comuns no atendimento da MFC na APS, como por exemplo as doenças vasculares periféricas (DVPs), compostas pelo acometimento da circulação nos sistemas arterial, venoso e/ou linfático. ${ }^{8}$

As DVPs podem decorrer de obstruções ou alteração no fluxo, incluindo a doença arterial obstrutiva periférica (DAOP) como o componente arterial mais comum, enquanto a insuficiência venosa crônica (IVC) é a doença decorrente da alteração mais prevalente no sistema venoso. ${ }^{8}$

Considerando a população adulta ocidental, a IVC acomete 10 a 20\% dos homens e 25 a $33 \%$ das mulheres, com progressão de acordo com o envelhecimento. ${ }^{9}$ A DAOP possui prevalência de 15 a $20 \%$ nas pessoas acima de 55 anos, em que grande parte desses são assintomáticos, cerca de 70 a $80 \% .^{10}$ Tais prevalências, somadas ao envelhecimento populacional verificado nas últimas décadas, levam a uma significativa morbidade, que justifica a importância do manejo competente destas doenças pelo médico de família e comunidade.

Dessa forma, os programas de residência em medicina de família e comunidade (PRMFCs), enquanto caminhos de capacitação profissional, possibilitam o aperfeiçoamento da prática médica, podendo melhorar indicadores de cuidado. ${ }^{11}$ Em João Pessoa, existem quatro PRMFCs, os quais inserem os médicos residentes em equipes de saúde da família de determinadas unidades. Os programas de residência médica são considerados padrão-ouro na formação de especialistas. ${ }^{12}$ No caso do PRMFC da Universidade Federal da Paraíba (UFPB), a gestão se dá em parceria com a secretaria de saúde do município de João Pessoa. O médico residente assume a função de médico na equipe e recebe suporte clínico e de gestão 
de um preceptor especialista em MFC, recebendo ainda tutoria de professores da UFPB. ${ }^{13}$ Este modelo consolidou-se e foi seguido pelas outras 3 escolas de medicina da região metropolitana de João Pessoa, constituindo-se um colegiado gestor único dos 4 PRMFCs com a secretaria de saúde do município, a fim de realizar as pactuações, definições e planejamento de forma unificada. Esta adoção de um modelo colegiado para os 4 programas, com participação direta da gestão municipal, trouxe grande consistência ao processo.

Um dos resultados desta forma de organização foi que, entre 2017 e 2018, houve uma importante expansão e consolidação dos PRMFCs dentro da rede de APS de João Pessoa. Esta foi fruto da articulação dos professores e profissionais da rede ligados à APS e à MFC. Além disso, é preciso destacar a política federal de ampliação de vagas com ofertas de bolsas de residência em MFC e o apoio da gestão municipal a tal proposta. Esta expansão levantou a necessidade de avaliar o impacto de tal iniciativa sobre a resolubilidade em APS. Para tanto, foram desenvolvidas, dentro dos PRMFCs, ações de capacitação junto aos médicos residentes e preceptores. Dentre estas, estão as ações voltadas para o cuidado de doenças venosas crônicas. Os residentes foram submetidos a treinamento para manejo de DVPs e disponibilizaramse alguns aparelhos Doppler vascular portátil para o cálculo do índice tornozelo-braquial (ITB) nas unidades de saúde da família (USFs) com maior população adscrita.

Neste sentido, considerando que as solicitações de exames complementares e encaminhamentos a especialistas focais podem dar indicativos sobre o nível de resolubilidade em APS, o presente estudo pretende avaliar as solicitações de ultrassonografias (USG) Doppler de vasos e os encaminhamentos a cirurgia vascular ou angiologia oriundos de USFs do município de João Pessoa/PB. Além disso, pretendese comparar a quantidade de solicitações com a população adscrita de cada USF, assim como avaliar se há diferenças no padrão das solicitações e encaminhamentos feitos a partir de unidades conveniadas com o PRMFC há mais de um ano, nas USFs com programas em fase de implantação durante o período analisado e naquelas USFs sem nenhum convênio com PRMFC. Finalmente, após análise descritiva e estatística dos dados, tais resultados foram discutidos à luz da P4 e das possíveis ações realizadas pelo médico de família no contexto das DVPs.

\section{MÉTODOS}

Trata-se de um estudo transversal feito a partir das solicitações de ultrassonografias Doppler de vasos e encaminhamentos para ambulatórios de cirurgia vascular ou angiologia provenientes de USFs do município de João Pessoa, entre janeiro de 2017 a agosto de 2018. Foram consultados bancos de dados secundários fornecidos pela regulação da secretaria municipal de saúde de João Pessoa. Por se tratar de consulta a bancos de dados secundários, termos de consentimento livre e esclarecidos não foram aplicados. A pesquisa foi submetida ao comitê de ética em pesquisa do centro de ciências médicas da UFPB sob o CAAE de número 02954918.0.0000.8069 e aprovada por este.

Os dados de solicitação de USG Doppler e de encaminhamentos para consulta em cirurgia vascular e angiologia foram obtidos com autorização da secretaria de saúde municipal, que utiliza o sistema de regulação do Ministério da Saúde SISREG versão 3 (SISREG III). Os dados de população adulta de cada equipe de saúde da família (eSF) foram obtidos mediante autorização, diretamente do setor da secretaria municipal de saúde responsável pela alimentação do sistema de informações da atenção básica (SISAB). 
Como os dados da regulação são por unidade de saúde da família (USF), enquanto os dados do SISAB são por equipes de saúde da família (eSF), tornou-se necessário identificar todas as USFs com mais de uma ESF (chamadas de USFs integradas) e somar as populações adscritas destas.

Para fim de análise, as USFs foram divididas em três grupos: unidades com médicos pertencentes ao PRMFC desde o início da coleta de dados, em janeiro de 2017; unidades que receberam médicos residentes em um momento posterior; e unidades sem médicos residentes. Com estes grupos foi realizada a análise descritiva e estatística quanto à taxa de requisição de USG Doppler e de encaminhamentos a médicos especialistas em cirurgia vascular ou em angiologia. Os dados não apresentaram padrão de distribuição normal no teste de normalidade de Shapiro-Wilk em nenhum dos três grupos. Por isso, nos resultados são apresentadas as medianas e, entre parênteses, o primeiro e terceiro quartis de distribuição dos dados. Por serem 3 grupos amostrais com dados não paramétricos (sem distribuição normal), escolheuse, para a comparação estatística, o teste não-paramétrico de Kruskall-Wallis. Foi considerado o nível de significância de 5\%. Utilizou-se o software SPSS 21.0 para construção do banco de dados e realização da análise descritiva e testes estatísticos.

\section{RESULTADOS}

Foram coletados dados de solicitações de USG Doppler de vasos realizadas nas USFs do município de João Pessoa no período de janeiro de 2017 a agosto de 2018, apresentados na Tabela 1. O número total de solicitações de USG Doppler de vasos em análise na central de regulação de João Pessoa foi de 3.918, sendo estes pedidos oriundos de um total de 90 USFs do município. Uma parte destas é integrada, havendo mais de uma eSF no mesmo imóvel (entre duas e quatro equipes), e uma parte é isolada, ou seja, com apenas uma eSF no imóvel. Embora a cidade de João Pessoa possua 201 eSFs cadastradas em atuação, a central de regulação não as identifica isoladamente, apenas as USFs, o que impede que se possa analisar os pedidos por equipe. Somadas, essas USFs contam com uma população adscrita maior de 18 anos de 332.819 usuários. A USF com maior número de solicitações $(n=194)$ foi a "Mudança de Vida". As unidades com menor número de solicitações foram USF Mandacaru VII (5) e USF Água Fria (5). A média de solicitações por USF no período considerado foi de 43,5 e de encaminhamentos a especialistas em cirurgia vascular ou angiologia foi 42,4 . Considerando a razão entre o número de exames solicitados e a população adulta adscrita, o município apresentou 1 Doppler para cada 85 habitantes adultos. A USF

Bairro das Indústrias III/Cidade Verde II, pertencente ao Distrito Sanitário I (DS-I) apresentou a maior razão de solicitação por adultos, sendo 1 Doppler para cada 24 adultos. A USF Integrada Rosa de Fátima, pertencente ao Distrito Sanitário III (DS-III), apresentou a menor razão, 1 Doppler para cada 717 adultos.

Outro ponto a ser considerado foi que o número de solicitações de USG obtido se refere a qualquer topografia vascular. Entretanto, pela conhecida prevalência da insuficiência venosa crônica na literatura, considerou-se a amostra representativa, em sua maioria, de ultrassonografias de membros inferiores. Quanto à taxa de encaminhamentos aos especialistas em cirurgia vascular, de modo semelhante, não se refere exclusivamente à doença venosa crônica incluindo uma fração de patologias menos frequentes, como aneurismas, estenoses e trombos. Quanto aos dados utilizados, obtidos de forma secundária na secretaria de saúde do município, não estavam sob o controle dos autores deste estudo. 
Tabela 1. Solicitações de USG Doppler de vasos em João Pessoa/PB de janeiro de 2017 a agosto de 2018.

\begin{tabular}{|c|c|c|}
\hline \multicolumn{2}{|c|}{ Quantidade total de USFs } & 90 \\
\hline \multicolumn{2}{|c|}{ Quantidade total de solicitações } & 3.918 \\
\hline \multicolumn{2}{|c|}{ População adscrita maior de 18 anos } & 332.819 \\
\hline USF com maior número de solicitações & Mudança de Vida & 194 \\
\hline USF com menor número de solicitações & Mandacaru VII/Água Fria & $5 / 5$ \\
\hline \multicolumn{2}{|c|}{ Média de solicitações por USF } & 43,5 \\
\hline Maior razão de solicitações por adultos & Bairro das Indústrias III/ Cidade Verde II & 0,0416 \\
\hline Menor razão de solicitações por adultos & Rosa de Fátima & 0,0014 \\
\hline
\end{tabular}

Fonte: Própria dos autores (2018).

Na Tabela 2, pode-se observar a análise descritiva e estatística das taxas de requisição de USG Doppler e de encaminhamentos a médicos especialistas em cirurgia vascular ou em angiologia. Houve diferença estatisticamente significativa no número de USG Doppler solicitadas nas USFs com PRMFC $(p<0,05)$, sendo esta taxa maior nas USFs com PRMFC do que nas sem PRMFC ou em implantação. Não houve diferença estatisticamente significativa entre as taxas de solicitação de USG Doppler das USFs sem PRMFC e das USFs com PRMFC em implantação. Tais dados estão ilustrados no Gráfico 1. Também houve diferença estatisticamente significativa da taxa de encaminhamentos ao especialista em cirurgia vascular ou angiologia nas USFs com PRMFC em relação aos demais grupos. Conforme é possível ver na Tabela 2 e no Gráfico 2, esta taxa foi menor nas USF com PRMFC, em relação às demais.

Tabela 2. Taxas de solicitação de USG de Doppler e encaminhamentos para angiologia e cirurgia vascular por população maior de 18 anos nos três grupos de USF.

\begin{tabular}{lccc}
\hline USF & $\mathbf{n}$ & $\begin{array}{c}\text { Solicitação de USG doppler/pop>18a. } \\
\text { Mediana (10-30 quartil) }\end{array}$ & $\begin{array}{c}\text { Encaminhamentos/pop>18a. } \\
\text { Mediana (10-3o quartil) }\end{array}$ \\
\hline com PRMFC implantado & 3 & $0,0225(0,0199-0,0225)$ & $0,00788(0,00553-0,00814)$ \\
PRMFC em implantação & 20 & $0,0094(0,00799-0,0138)$ & $0,0115(0,00944-0,0169)$ \\
\hline sem PRMFC & 67 & $0,0114(0,00747-0,0166)$ & $0,012(0,00798-0,00814)$ \\
\hline
\end{tabular}

Gráfico 1. Taxas de solicitação de USG Doppler por população adscrita maior de 18 anos.

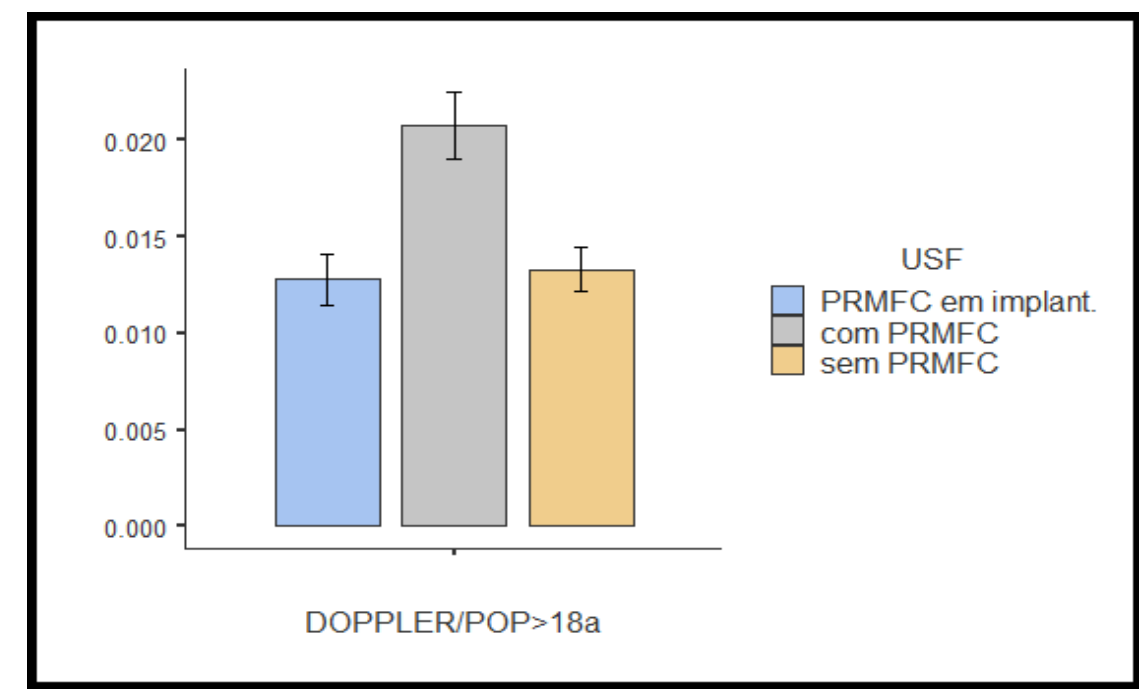

Legenda: A altura das barras indica a mediana e o segmento de reta indica o $1^{\circ} \mathrm{e} 3^{\circ}$ quartis. 
Gráfico 2. Taxas de encaminhamento para cirurgia vascular e angiologia por população adscrita maior de 18 anos.

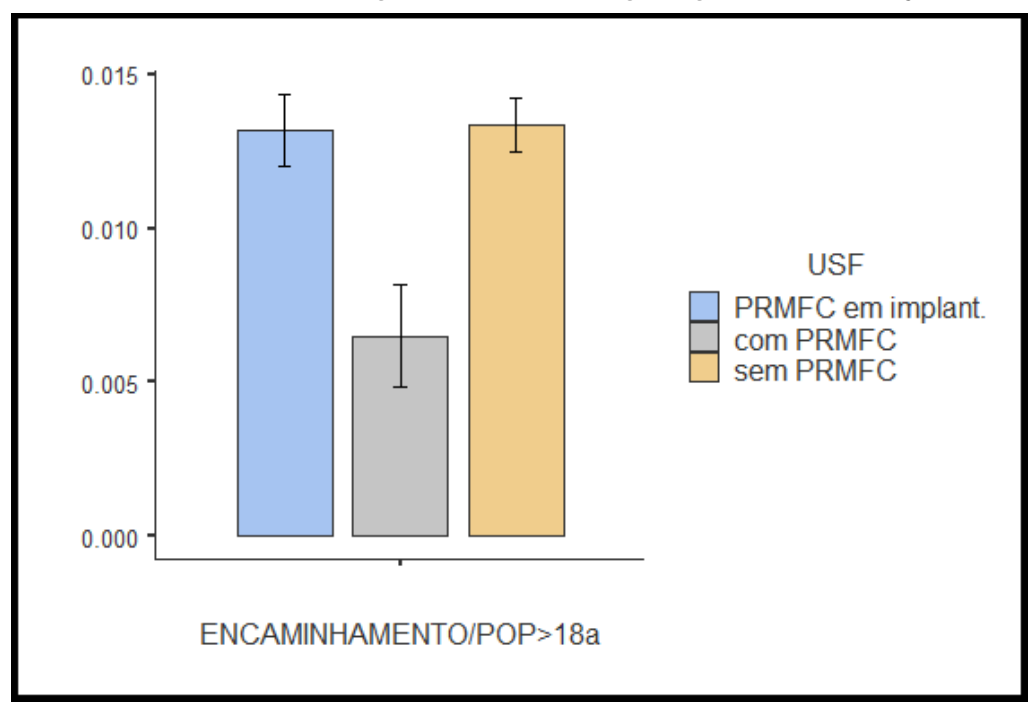

Legenda: A altura das barras indica a mediana e o segmento de reta indica o $1^{\circ} \mathrm{e} 3^{\circ}$ quartis.

\section{DISCUSSÃO}

Os resultados do estudo sugerem que os médicos residentes solicitam mais exames que os médicos não residentes, mas, por outro lado, apresentam menor taxa de encaminhamento aos especialistas. Este achado pode sugerir que o programa de residência em medicina de família e comunidade leva a um aumento da resolubilidade dos médicos, seja pela qualidade da supervisão do preceptor, seja pelo perfil dos profissionais que buscam o programa. Entretanto, novos estudos com delineamentos mais específicos devem ser realizados para a investigação destas hipóteses.

A diminuição dos encaminhamentos aos especialistas focais pode ser uma característica do residente em MFC, mais permeáveis e sensibilizados ao ensino da prevenção quaternária e, provavelmente, relacionada à longitudinalidade do cuidado, melhorando o acesso daqueles pacientes que precisam de atendimento nos demais níveis de atenção, diminuindo o custo gerado pelos atendimentos em serviços especializados sem indicação clara e os danos próprios das intervenções médicas desnecessárias. ${ }^{14} \mathrm{~A}$ confirmar-se esta hipótese, a atitude do residente iniciante poderia não implicar em reduzir custos imediatos com exames complementares, mas teria potencial de gerar tal efeito a longo prazo, visto que residentes mais experimentados, sob supervisão de preceptores qualificados, desenvolvem as competências necessárias para adequação da solicitação de exames. A utilização dos exames complementares de maneira adequada à melhor conduta médica protege a população do risco de hipermedicalização e de intervenções invasivas ao gerar menos encaminhamentos aos procedimentos com baixos graus de evidência. ${ }^{15,16}$

Os residentes, ao longo do processo de formação, são expostos a situações biopsicossociais delicadas, trazendo à tona o conceito de prevenção quaternária (P4). Esta, potencializa o olhar crítico sobre a atividade médica no campo da prática com a finalidade primeira e última de não prejudicar. A P4 envolve o imperativo moral de monitoramento rigoroso do próprio médico, o que, na figura do residente, ajuda o preceptor do PRMFC a avaliá-lo enquanto um bom consultor que trata das pessoas, colocando-as no centro do cuidado. ${ }^{17,18}$ 
O menor número de encaminhamentos para especialistas focais pode ser uma causa de redução de intervenções desnecessárias ou hipermedicalização. ${ }^{19}$ Neste sentido, os embasamentos clínico e crítico do médico da APS devem ser adequados diante das patologias encontradas no seu cotidiano, cujos diagnósticos são eminentemente clínicos, como nas doenças vasculares periféricas. Deste modo, enfermidades como IVC e DAOP se enquadram nessa categoria, podendo ser diagnosticadas sem a necessidade de muitos recursos. Sendo assim, é possível correlacionar a P4 e as habilidades clínicas do médico, uma vez que o profissional sendo habilitado a manejar as doenças do ponto de vista clínico implica em menor taxa de encaminhamentos e até mesmo condutas iatrogênicas.

Tais conceitos corroboram o fortalecimento da APS, a fim de aumentar sua ação preventiva ante procedimentos desnecessários, principalmente nas condições clínicas mais comuns, como a insuficiência venosa crônica. O tratamento da IVC é baseado no quadro clínico, sendo o MFC capaz de conduzir os casos sem equipamentos complexos através de orientações simples, tais como: mudanças dietéticas, exercícios físicos, diminuição dos fatores de risco modificáveis e do uso de meias compressivas ou medicações vasoativas quando necessário. ${ }^{21}$

Em estudo realizado no Rio de Janeiro, em 2016, percebeu-se que a inserção de PRMFC nas unidades de saúde impõe melhoria da qualidade dos profissionais, inclusive não-médicos, assim como da organização do trabalho e da oferta de serviços e vínculo dos pacientes. Logo, almeja-se que as demais USFs do município, as quais receberam o PRMFC após o início do presente estudo, sigam o padrão de aperfeiçoamento apresentado, evoluindo com resultados semelhantes no futuro. ${ }^{17}$

Para potencializar benefícios e atenuar danos e custos, é necessário organizar estratégias para cada perfil, considerando a complexidade e a disponibilidade das intervenções. O leque de ofertas preventivas reforça a necessidade de uma escolha racional, levando em conta o risco absoluto global, as preferências e os recursos do paciente. ${ }^{22}$

A P4 torna mais vigorosas as capacidades críticas e epidemiológicas dos médicos de família e comunidade, características nem sempre contempladas na graduação em medicina, necessitando de reforço massivo na residência, porque estão em queda dentre os médicos em geral. A medicina, cada vez mais enrijecida em protocolos e padronizações de tratamentos, segue com interpretações simplórias, por vezes medíocres, pouco percebendo que cada pessoa adoece à sua maneira. Isto coloca os doentes como um grupo homogêneo, indo no sentido oposto da abordagem centrada no indivíduo, que leva em consideração suas peculiaridades e o eixo existencial-social-psicológico em que está inserido. ${ }^{23}$

\section{CONCLUSÃO}

A residência segue como padrão-ouro para formação de especialistas, visto imergir os profissionais no serviço de saúde. O menor número de encaminhamentos desnecessários ou fúteis aos especialistas focais corroborou com o compromisso da qualidade da assistência ofertada pela residência médica e com a longitudinalidade dos cuidados ofertados. Estudos quantitativos inovadores podem ser aplicados no futuro, quando as USFs terão maior consolidação do PRMFC, podendo apresentar resultados semelhantes, quiçá mais robustos.

O desafio também é válido aos estudos qualitativos, visto que as doenças vasculares com potencial ulcerativo são estigmatizantes e albergam experiências do adoecimento peculiares. Nesse ínterim, tal 
formato de pesquisa possui capilaridade para fortalecer as competências almejadas à formação do especialista em MFC, no que se refere à resolutividade ampliada e aos desafios inerentes à APS. No início da especialização, o primeiro ano do programa, existe a possibilidade de maior número de solicitações de exames complementares; todavia, as USFs com mais experiência na condução do PRMFC podem auxiliar melhor os residentes, sensibilizando-os no que diz respeito à $\mathrm{P} 4$, o que pode vir a diminuir tal quantitativo, abrindo espaço para um cuidado longitudinal ainda mais robusto.

\section{Colaboradores}

Concepção e delineamento do estudo: AT, DM, ES, KF, LM, MP, MM. Aquisição, análise ou interpretação dos dados: ES, KF. Redação preliminar: AT, DM, KF, LM, MP, MM. Revisão crítica da versão preliminar: DM, ES. Todos os autores aprovaram a versão final e concordaram em prestar contas sobre todos os aspectos do trabalho.

\section{Conflito de interesse}

Não há conflitos de interesses.

\section{REFERÊNCIAS}

1. Norman $A H$, Tesser $C D$. Prevenção quaternária na atenção primária à saúde: uma necessidade do Sistema Único de Saúde. Cad Saúde Pública. 2009;25(9):2012-20. DOI: https://doi.org/10.1590/S0102-311X2009000900015

2. Oliveira CC, Reis A. Questões epistemológicas e bióticas da prevenção quaternária.Physis [Internet].2012; [citado 2020 Jun 10];22(4):1485502. Disponível em: https://www.scielo.br/pdf/physis/v22n4/a12v22n4.pdf DOI: https://doi.org/10.1590/S0103-73312012000400012

3. Pizzanelli M, Almenas M, Quirós R, Pineda C, Cordero E, Taureaux N, et al. Quaternary prevention: medical ethics, evaluation and efficiency in the health systems. Rev Bras Med Fam Comunidade. 2016;11(Supl 2):75-85. DOI: http://dx.doi.org/10.5712/rbmfc11(0)1388 DOI: https://doi.org/10.5712/rbmfc11(0)1388

4. Almenas M, Cordero E, Andrés C, Muñoz E, Rojas ML, Salvatierra E, et al. Quaternary prevention: how to do, how to teach. Rev Bras Med Fam Comunidade. 2018;13(Supl 1):69-83. DOI: https://doi.org/10.5712/rbmfc13(1)1853

5. Pérez-Fernández M, Gérvas J. El efecto cascada: implicaciones clínicas, epidemiológicas y éticas. Med Clin (Barc). 2002;118(2):65-7. DOI: https://doi.org/10.1016/S0025-7753(02)72283-5

6. Castro Filho ED. Telessaúde em apoio à atenção primária à saúde no Brasil. Rev Bras Med Fam Comunidade [Internet].2007; [citado 2020 Jun 08]; 11(3):210-5. Disponível em: https://www.rbmfc.org.br/rbmfc/article/view/227/180 DOI: https://doi.org/10.5712/rbmfc3(11)227

7. Janaudis MA. Princípios da medicina de família: quatro pilares que definem sua identidade. Mundo Saúde [Internet]. 2010; [citado 2020 Jun 07]; 34(3):300-10. Disponível em: http://www.saocamilo-sp.br/pdf/mundo_saude/77/300a310.pdf DOI: https://doi.org/10.15343/01047809.20103300310

8. Costa IF, Burihan E. Manifestações clínicas das doenças vasculares periféricas. In: Nectoux JE, ed. Doença vascular periférica: métodos diagnósticos não invasivos. Rio de Janeiro: Revinter; 1994. p. 1-9.

9. Pereira AH, Pereira AA. Doenças venosas dos membros inferiores. In: Duncan BB, Schmidt MI, Giugliani ERJ, eds. Medicina ambulatorial: condutas de atenção primária baseadas em evidências. 4a ed. Porto Alegre: Artmed; 2013.

10. Norman PE, Eikelboom JW, Hankey GJ. Peripheral arterial disease: prognostic significance and prevention of atherothrombotic complications. Med J Aust [Internet].2004; [ citado 2020 Jun 08]; 181(3):150-4. Disponível em: https://pubmed.ncbi.nlm.nih.gov/15287833/ PMID: 15287833 DOI: https://doi.org/10.5694/j.1326-5377.2004.tb06206.x

11. Castro VS, Nóbrega-Therrienll SM. Residência de medicina de família e comunidade: uma estratégia de qualificação. Rev Bras Educ Méd [Internet]. 2009; [citado 2020 Jun 10]; 33(2):211-20. Disponível em: https://www.scielo.br/pdf/rbem/v33n2/08.pdf DOI: https://doi. org/10.1590/S0100-55022009000200008 
12. Bevilacqua RG, Sampaio SAP. As especializações: histórico e projeções. In: Negri B, Faria R, Viana ALD, orgs. Recursos humanos em saúde: política, desenvolvimento e mercado de trabalho. Campinas: Editora Unicamp; 2002. p. 33-90.

13. Soares RS, Oliveira FP, Melo Neto AJ, Barreto DS, Carvalho ALB, Sampaio J, et al. Residência em medicina de família e comunidade: construindo redes de aprendizagens no SUS. Rev Bras Med Fam Comunidade. 2018;13(40):1-8. DOI: https://doi.org/10.5712/ rbmfc13(40)1629

14. Elias E, Magajewski F. A atenção primária à saúde no sul de Santa Catarina: uma análise das internações por condições sensíveis à atenção ambulatorial, no período de 1999 a 2004. Rev Bras Epidemiol [Internet]. 2008; [citado 2020 Jun 08]; 11(4):633-47. Disponível em: https://www.scielo.br/pdf/rbepid/v11n4/10.pdf DOI: https://doi.org/10.1590/S1415-790X2008000400011

15. Gross DMP, Camacho ACLF, Lage LR, Daher DV, Mota CP. Prevenção quaternária na gestão da atenção à saúde: revisão integrativa. Rev Enferm UFPE Online [Internet]. 2016; [citado 2020 Jun 11]; 10(4):3608-19. Disponível em: https://pesquisa.bvsalud.org/enfermeria/ resource/pt/bde-29974

16. Mangin D, Heath I. Multimorbidity and quaternary prevention (P4). Rev Bras Med Fam Comunidade [Internet]. 2015; [citado 2020 Jun 08]; 10(35):1-5. Disponível em: https://www.rbmfc.org.br/rbmfc/article/view/1069 DOI: https://doi.org/10.5712/rbmfc10(35)1069

17. Jamoulle M. Quaternary prevention, as answer of family doctors to overmedicalization. Int J Health Policy Manag [Internet]. 2015; [citado 2020 Jun 07]; 4(2):61-4. Disponível em: https://www.ijhpm.com/article_2950.html PMID: 25674569 DOI: https://doi.org/10.15171/ ijhpm.2015.24

18. Tesser CD. Prevenção quaternária para a humanização da Atenção Primária à Saúde. Mundo Saúde [Internet]. 2012 Jul/Set; [citado 2020 Jun 10]; 36(3):416-26. Disponível em: https://pesquisa.bvsalud.org/bvsms/resource/pt/mis-36725

19. Moynihan R, Doust J, Henry D. Preventing overdiagnosis: how to stop harming the healthy. BMJ [Internet]. 2012 ; [citado 2020 Jun 11]; 344:e3502. Disponível em: https://www.bmj.com/content/344/bmj.e3502 PMID: 22645185 DOI: https://doi.org/10.1136/bmj.e3502

20. Ribeiro MTAM, Fiuza TM, Barros HM, Montenegro Jr R. Doenças do sistema venoso. IN: Gusso G, Lopes JMC, Dias LC. Tratado de medicina de família e comunidade. 2a ed. Porto Alegre: Artmed; 2019, p. 1403-1412..

21. Silva MC, Cabral ALS, Barros Junior N, Castro AA, Santos MERC. Diagnóstico e tratamento da doença venosa crônica. J Vasc Bras [Internet]. 2005; 4(3 Supl 2):185-93. Disponível em: http://www.saudedireta.com.br/docsupload/1340062323Arquivo_1.pdf

22. Ministério da Saúde (BR). Secretaria de Atenção à Saúde. Departamento de Atenção Básica. Cadernos de Atenção Básica. Prevenção clínica de doença cardiovascular, cerebrovascular e renal crônica. Brasília (DF): Ministério da Saúde; 2006.

23Norman AH, Tesser CD. Prevenção quaternária: as bases para sua operacionalização na relação médico-paciente. Rev Bras Med Fam Comunidade. 2015;10(35):1-10. DOI: http://dx.doi.org/10.5712/rbmfc10(35)1011 